\title{
Portée des quantifieurs et ordres des mots en coréen
}

Jung-Sup Bak

\section{(2) OpenEdition \\ Journals}

Édition électronique

URL : http://journals.openedition.org/rlv/1218

DOI : $10.4000 /$ rlv. 1218

ISSN : 1958-9239

Éditeur

Presses universitaires de Vincennes

Édition imprimée

Date de publication : 1 mai 1999

Pagination : 157-178

ISSN : 0986-6124

\section{Référence électronique}

Jung-Sup Bak, "Portée des quantifieurs et ordres des mots en coréen ", Recherches linguistiques de Vincennes [En ligne], 28 | 1999, mis en ligne le 16 septembre 2005, consulté le 22 avril 2019. URL http://journals.openedition.org/rlv/1218; DOI : 10.4000/rlv.1218 


\title{
Jung-Sup BAK \\ Université Sogang
}

\section{PORTÉE DES QUANTIFIEURS ET ORDRES DES MOTS EN CORÉEN *}

\begin{abstract}
RÉSUMÉ
Cet article vise à décrire la portée des quantifieurs dans diverses constructions à quantifieurs multiples en coréen. Pour ce faire, il adopte la théorie de Diesing qui permet d'expliquer de nombreux phénomènes de quantification dans diverses langues. Il propose par ailleurs une analyse nouvelle d'un déplacement dit « M(iddle)-scrambling » dans des langues comme le coréen. Depuis longtemps, il a été admis dans la littérature que le sujet se comporte comme une exception au regard de M-scrambling. Mais cette idée couramment admise est rejetée dans ce travail. Il est démontré ici que Mscrambling est un déplacement vers une position hors de $v \mathrm{P}$ qui concerne tous les syntagmes non prédicatifs. Cette analyse de M-scrambling, combinée avec la théorie de Diesing, permet de rendre compte correctement des faits de quantification en coréen.
\end{abstract}

\section{MOTS-CLÉS}

Portée des quantifieurs, quantifieur fort, quantifieur faible, M-scrambling, focalisation, reconstruction, Diesing, QR, présupposition, interprétation spécifique, interprétation variable, coréen, ambiguïté. 


\section{Introduction}

Depuis longtemps, l'ambiguïté des phrases comme les suivantes a été décrite et commentée par les logiciens et les linguistes :

a. Someone loves everyone.

b. Everyone loves someone.

On peut avoir au moins deux lectures pour chacune des phrases (1) selon la portée des quantifieurs ${ }^{1}$. Soit l'univers de discours où il existe trois personnes $\mathrm{A}, \mathrm{B}, \mathrm{C}$. Lorsque «someone » a la portée large, les phrases (a) et (b) signifient respectivement qu'une des trois personnes aime toutes les autres, et qu'une des trois personnes est aimée par toutes les autres. Si en revanche, « someone » a la portée étroite, les phrases voudraient dire respectivement que chacune des trois personnes est aimée par quelqu'un d'autre, et que chacune des trois personnes aime quelqu'un d'autre.

Cela dit, d'après Huang (1982), cette ambiguïté souvent observée dans des langues comme l'anglais, ne se retrouve pas en chinois, et dans l'équivalent chinois de (1b), c'est toujours « everyone » qui a la portée large. Certains linguistes ont déjà essayé, chacun à sa manière, de rendre compte de cette différence entre des langues comme l'anglais et des langues comme le chinois. Mais la question de portée des quantifieurs dans celles-ci semble encore loin d'être élucidée de façon satisfaisante. Je voudrais donc avancer, dans ce travail, une analyse pour les faits concernant la portée des quantifieurs en coréen, langue souvent censée se comporter comme le chinois en ce qui concerne la portée des quantifieurs, en me basant essentiellement sur la théorie de Diesing (1992) ${ }^{2}$.

Pour ce faire, je commencerai par passer brièvement en revue quelques théories de la quantification dans la prochaine section. Ensuite, en section 2, je décrirai le phénomène dit $\mathrm{M}$-scrambling, qui constitue une des caractéristiques du coréen. En section 3, il sera démontré que la théorie de Diesing et les propriétés de M-scrambling décrites en section 2 prédisent correctement la portée des quantifieurs en coréen. Enfin, dans la section 4, je présenterai quelques idées spéculatives dont j'aurai besoin pour réconcilier les notions adoptées dans ce travail avec la Théorie Minimaliste de Chomsky (1995), mais qui ne sont pas établies solidement du point de vue empirique.

\section{Quelques théories de la quantification}

Dans certaines théories de la quantification, il est admis que la trace laissée par la montée de quantifieur (désormais QR) ou des déplacements semblables joue un rôle dans la détermination de la portée des quantifieurs. En d'autres termes, ces théories prennent en compte, pour le calcul de celle-ci, les chaînes formées du quantifieur et de ses traces. En revanche, dans d'autres théories, la portée des quantifieurs se détermine uniquement en fonction de leur position en 
FL, c'est-à-dire que les traces n'y jouent aucun rôle. Dans cette section, je me propose de regarder de près ces deux types de théories pour savoir lequel permet de décrire le plus correctement la portée de QP en coréen. Mais sans prétendre donner une classification complète des théories de la quantification, je passerai rapidement en revue les théories de Aoun et Li (1989) et de Diesing (1992), en les considérant respectivement comme représentatives des deux types de théorie.

\subsection{Aoun \& Li (1989)}

La particularité du chinois observée par Huang (1982) concernant la portée des quantifieurs dans certaines constructions a attiré l'attention de nombreux linguistes dont Aoun et Li (1989; désormais AL) qui en proposent une explication systématique dans le cadre de la Théorie GB. Considérons les phrases suivantes :

$$
\begin{aligned}
& \text { Meigeren dou xihuan yige nuren } \\
& \text { everyone all like one woman [= (1a) de AL] } \\
& {[=(1 b)] \text { Everyone loves someone }}
\end{aligned}
$$

À la différence de (3), son équivalent approximatif chinois est sans ambiguïté : en (2), seul le QP sujet peut avoir la portée large, alors qu'en (3), chacun des deux QP peut l'avoir. Pour rendre compte de cette différence, AL supposent que dans des langues comme le chinois où la pauvreté morphologique de Infl ne rend pas possible la montée du sujet, le sujet de la phrase doit être engendré directement en Spec de IP, tandis que dans des langues comme l'anglais, conformément à l'Hypothèse du sujet interne à VP, le sujet est engendré en Spec de VP, puis monté en syntaxe en Spec de IPpour des raisons casuelles. ALproposent par ailleurs les principes suivants :

(4) Exigence sur le liage minimal

Une variable doit être liée par le lieur-A' potentiel le plus local

Principe de portée

Un quantifieur A a la portée sur un quantifieur B si A c-commande un membre de la chaîne contenant B

Selon (4), dans des langues comme l'anglais, le QP objet pourra, lorsque QR s'y applique, s'adjoindre à VP, et donc c-commander la trace du sujet laissée dans sa position d'origine, c'est-à-dire Spec de VP, car celle-ci n'est pas une variable. Or la trace du QP objet est toujours c-commandée par le sujet. Par conséquent, l'ambiguïté des phrases anglaises comme (1a) ou (1b) découle du principe (5). En revanche, AL soutiennent que dans des langues comme le chinois où le sujet est engendré hors de VP, il est impossible pour le QPobjet, même lorsqu'il est adjoint à VP par QR, de c-commander le sujet ou sa trace s'il se déplace lui-même plus haut. Par ailleurs, dans ces langues, (4) exclut le QR du QP objet par-dessus la trace du QP sujet laissée en Spec de IP au terme de QR, car celle-ci est une 
variable. Les auteurs concluent que dans les phrases chinoises à quantifieurs multiples, le QP objet ne peut c-commander aucun membre de la chaîne du QP sujet, et que selon (5), on s'attend à l'absence d'ambiguïté dans des phrases comme (2).

Ainsi, l'analyse de AL semble décrire assez correctement la différence entre l'anglais et le chinois. Mais elle ne paraît pas aussi adéquate pour décrire les faits de quantification dans des langues comme le coréen. Considérons les exemples coréens suivants ${ }^{3}$ :

(6) ôt'ôn salam-i motu-lîl mannas'ta

quelqu'un-Nom tous-Acc rencontra

'Quelqu'un a rencontré tout le monde.'

motu-ka ôt'ôn salam-îl mannas'ta

tous-Nom quelqu'un-Acc rencontra

'Tout le monde a rencontré quelqu'un.'

En (6), on observe l'absence d'ambiguïté comme le prédirait l'analyse de AL: la seule interprétation possible de la phrase serait quelque chose comme « $\mathrm{X}$ a rencontré A, B, C... ». Mais la phrase (7), tout comme sa traduction française, est bien ambiguë : en gros, elle signifie à la fois « $\mathrm{X}, \mathrm{Y}, \mathrm{Z} \ldots$ ont rencontré $\mathrm{A}$ » et « $\mathrm{X}$ a rencontré $\mathrm{A}, \mathrm{Y}$ a rencontré $\mathrm{B}$, ainsi de suite... " ${ }^{4}$. Dans l'hypothèse où en coréen, le sujet est engendré en Spec de IP comme le soutiennent AL pour le chinois, l'ambiguïté de (7) sera mystérieuse. Par ailleurs, si l'on pose qu'en coréen le sujet est engendré en Spec de VP (ou $v \mathrm{P}$ si on prend des termes plus modernes), puis monté en Spec de IP, alors l'absence d'ambiguïté dans la phrase (6) sera difficile à expliquer, car la structure de celle-ci serait exactement identique à celle de (1a) qui est ambiguë. Il semble donc que la prédiction de $\mathrm{AL}$ concernant (6) ou bien celle concernant (7) est nécessairement incorrecte, quelle que soit la position d'origine du sujet qu'on pose pour le coréen.

Il semble d'autre part que le paramètre concernant la position du sujet proposée par AL n'est pas justifiée du point de vue morphologique. Les auteurs supposent qu'en chinois, Infl n'est pas assez riche pour rendre possible la montée du sujet depuis VP jusqu'à Spec de IP, ce qui conduit les langues en question à adopter la stratégie de génération directe du sujet en Spec de IP ${ }^{5}$. Cela dit, en coréen ou en japonais, même si dans ces langues, tout comme en chinois, l'absence d'ambiguïté est observée pour des phrases du genre (1a), Infl ne paraît pas aussi pauvre qu'en chinois : on peut citer comme éléments de Infl les morphèmes de Temps et d'Aspect, et le morphème honorifique qui est le plus souvent considéré comme une variante de morphème d'Accord. Ainsi, l'analyse de $\mathrm{AL}$ ne semble pas adéquate pour rendre compte des faits dans des langues comme le coréen, et pour ces derniers, il faudrait une explication qui soit différente de celle de AL. 


\subsection{Diesing (1992)}

Si l'analyse de AL représente la théorie en termes de chaîne QP, on pourrait considérer que les travaux de May et de Diesing représentent une théorie qui ne tient compte que des têtes de chaîne QP. Je présenterai donc dans ce qui suit quelques idées de ces derniers auteurs.

Pour rendre compte de l'ambiguïté dans des phrases comme celles de (1), May (1977) propose une solution formelle en termes de QR. Elle consiste grosso modo à poser que les $\mathrm{QP}$ doivent obligatoirement monter vers une position-A', et que la portée de chaque QP se détermine par sa position syntaxique en FL. Lorsqu'en (1a), par exemple, « someone » se trouve plus haut que « everyone » au terme de l'opération $\mathrm{QR}$, la portée large sera attribuée à « someone », et dans le cas contraire, « someone » aura la portée étroite. Ainsi, dans la théorie de May (1977), des deux QP contenus dans une même phrase comme celles de (1), c'est celui occupant une position plus élevée en FL qui a la portée large.

Diesing (1992) propose une modification de la théorie de May dans son ouvrage sur les expressions indéfinies. Elle pose d'abord, adoptant à sa manière Heim et Kamp, qu'en FL, une phrase est divisée essentiellement en deux parties : celle regroupant les éléments qui relèvent de la présupposition (" clause restrictive » dans la terminologie de Diesing) et celle où se trouvent les autres éléments («portée nucléaire » dans la terminologie de Diesing). Diesing avance ensuite l'Hypothèse de Mapping ${ }^{6}$ pour exprimer l'idée que cette distinction d'ordre sémantico-pragmatique est représentée en syntaxe, plus précisément en FL. Ainsi, d'après elle, les éléments présupposés qui sont initialement engendrés sous VP ${ }^{7}$ doivent se déplacer hors de VP par QR exactement comme dans la théorie de May. Mais, à la différence de May, elle soutient crucialement que QR ne s'applique pas aux éléments non présupposés : les non présupposés doivent se (re)trouver sous VPen FLmême s'ils ont été déplacés hors de VPpar mouvementA avant $\mathrm{FL}^{8}$. Elle distingue par ailleurs, à la suite de Milsark (1974), deux types de QP : les QP dits « forts » qui relèvent toujours de la présupposition, et les QP «faibles » qui n'en relèvent pas toujours ${ }^{9}$. Par conséquent, dans cette théorie, QR s'applique obligatoirement aux QP forts, et optionnellement aux QP faibles.

La théorie de Diesing permet, me semble-t-il, de comprendre pourquoi QR ou un processus semblable doit avoir lieu dans les langues naturelles. Il est raisonnable de poser que le présupposé doit de toute façon se distinguer du non présupposé dans la composante interprétative Conceptuelle-Intentionnelle, et que les informations nécessaires à cette distinction interprétative y sont transmises de la représentation en FL. Diesing semble fournir une raison d'être à QR, en proposant précisément que QR est un moyen de placer les éléments présupposés dans un domaine différent de celui du non présupposé, rendant ainsi la phrase interprétable dans la composante C-I.

$\mathrm{Du}$ point de vue empirique, la théorie de Diesing rend compte d'un nombre considérable de données qui échappent à la théorie de May. Par exemple, Diesing prend en considération, non seulement les quantifieurs universels et 
existentiels, mais tout un ensemble d'indéfinis, dont les noms pluriels nus qui ne sont pas étudiés par May ${ }^{10}$. Par ailleurs, Diesing note que sa théorie prédit correctement l'existence de trois interprétations dans des phrases comme (8), tandis que la théorie de May n'en décrit que deux :

Every cellist played some variations.

D’après May (1977), (8) peut être interprété seulement de deux manières différentes, en fonction de la portée assignée aux deux QP, c'est-à-dire (i) some $>$ every et (ii) every > some. Mais Diesing soutient que l'interprétation (ii) doit être elle-même subdivisée en deux suivant la (non-) présuppositionnalité de « some variations », alors qu'en (i), le DP objet « some variations » est toujours présupposé. Voici les représentations correspondant aux trois interprétations de (8), données par Diesing (1992, p. 66) ${ }^{11}$ :
a. $\quad\left[\right.$ Every $_{\mathrm{x}}[$ cellist $(\mathrm{x})]$ Some $_{\mathrm{y}}[$ variation $(\mathrm{y})] \mathrm{x}$ played $\left.\mathrm{y}\right]$
b. $\quad\left[\right.$ Every $_{\mathrm{x}}$ [cellist $\left.(\mathrm{x})\right] \exists_{\mathrm{y}}$ variation $(\mathrm{y}) \wedge \mathrm{x}$ played $\left.\mathrm{y}\right]$
c. $\left[\right.$ Some $_{\mathrm{y}}[\text { variation }(\mathrm{y})]^{\mathrm{E}}$ Every $_{\mathrm{x}}[$ cellist $(\mathrm{x})] \mathrm{x}$ played $\left.\mathrm{y}\right]$

Dans son système, « every » un QP fort doit figurer obligatoirement hors de VP, alors que «some » un QPfaible peut à la fois se déplacer hors de VPet rester sous VP. Par conséquent, « some variations », non présupposé, reste sur place, mais s'il est présupposé, ce syntagme se déplace hors de VP, finissant éventuellement dans une position même plus élevée que « every cellist». Ainsi, la théorie de Diesing prédit correctement les trois interprétations pour (8), alors que celle de May n'est pas à même de distinguer deux interprétations avec la portée étroite pour « some », qui sont représentées dans (9a) et (9b).

En bref, la théorie de Diesing semble avoir des avantages tant conceptuels qu'empiriques sur la théorie de la quantification présentée par May (1977). Je démontrerai dans les deux sections qui suivent que la théorie de Diesing, combinée avec une théorie adéquate de la structure de la phrase, permet de rendre compte des faits de quantification dans les constructions à quantifieurs multiples en coréen, notamment en résolvant les problèmes qui se posent dans l'analyse de AL.

\section{M-scrambling et la position du sujet en coréen ${ }^{12}$}

Il est possible dans des langues comme le coréen ou le japonais, que l'objet se déplace par-dessus le sujet, c'est-à-dire à sa gauche. Alors que ce phénomène, souvent appelé « $M$ (iddle)-scrambling » (désormais M-scr), est décrit et discuté dans de nombreux travaux, beaucoup de questions continuent toujours à se poser à ce sujet. Dans cette section, je me propose d'en considérer quelques-unes comme les suivantes : 
(A) Quels sont les éléments qui peuvent se déplacer par M-scr ?

(B) Est-il obligatoire ou optionnel ?

(C) Est-ce un mouvement vers une position A ou A'?

\subsection{M-scr et le sujet}

Commençons par (A). En coréen, tout constituant non prédicatif de la phrase peut se mettre en tête de phrase comme le montrent les exemples suivants :
a. Pierre-ka Marie-eke phyônci-lîl ponäs'ta
-Nom -Dat lettre-Acc envoya
'Pierre a envoyé une lettre à Marie.'
b. Marie-eke Pierre-ka phyônci-lîl ponäs'ta
-Dat -Nom lettre-Acc envoya
c. phyônci-lîl Pierre-ka Marie-eke ponäs'ta
lettre-Acc
-Nom
-Dat envoya
a. Pierre-ka ton-îl cigap-e nôhôs'ta
-Nom argent-Acc bourse-Loc mit
'Pierre a mis l'argent dans sa bourse.'
b. ton-îl Pierre-ka ikap-e nôhôs'ta argent-Acc -Nom bourse-Loc mit
c. cikap-e Pierre-ka ton-îl nôhôs'ta bourse-Loc -Nom argent-Acc mit

Si on définit M-scr comme un changement dans l'ordre relatif entre le sujet et un autre élément de la phrase, les phrases $(10 b, c)$ et $(11 b, c)$ seront toutes considérées comme des instances de M-scr. Dans ces exemples sont déplacés : l'objet direct, l'objet indirect, le circonstant. Ainsi défini, M-scr s'appliquerait aux éléments non prédicatifs de la phrase sauf au sujet ${ }^{13}$. En fait, la plupart des linguistes admettent implicitement ou explicitement que M-scr ne concerne pas le sujet. La question se poserait immédiatement de savoir pourquoi le sujet est exclu de ce déplacement, pourquoi le sujet se comporte comme une exception au regard de M-scr. Pour apporter une réponse à cette question, on pourrait chercher un trait morphologique, syntaxique ou sémantique qui puisse être assigné à n'importe lequel des éléments non prédicatifs de la phrase à l'exception du sujet et supposer que c'est ce trait qui motive M-scr. Mais à ma connaissance, l'existence d'un tel trait n'a jamais été montrée dans les études linguistiques actuelles. La recherche dans cette direction me semble donc peu prometteuse. L'autre voie de recherche serait une modification de la définition habituelle de $\mathrm{M}$-scr : celui-ci serait redéfini comme un déplacement vers une position syntaxique déterminée, plus élevée que celle du sujet, par exemple, comme adjoint à $\mathrm{IP}^{14}$. Ainsi redéfini, Mscr concernera aussi le sujet : il pourra, de même que les autres constituants non prédicatifs de la phrase, se déplacer par M-scr vers la gauche. Comme cette redéfinition présente un avantage conceptuel considérable sur la définition 
habituelle, il conviendrait d'adopter celle-là, à moins que l'exclusion du sujet ne soit justifiée par un argument indépendant.

Notons au passage que certains linguistes ont déjà parlé du mouvement-A' du sujet dans des langues comme le coréen, en le considérant comme une topicalisation ou une focalisation, alors que peu de linguistes ont essayé de le mettre en rapport avec les autres déplacements en tête de phrase, qui eux sont communément considérés comme M-scr ${ }^{15}$. Or ces derniers aussi peuvent avoir souvent un effet de topicalisation ou de focalisation. Il n'y aurait donc aucune raison, me semble-t-il, de mettre à part ledit mouvement-A' du sujet. En admettant plutôt que ce dernier et les autres déplacements en tête de phrase relèvent d'un même phénomène ${ }^{16}$, on pourrait à la fois faire l'économie d'une confusion inutile et simplifier considérablement la théorie.

\subsection{Le caractère obligatoire de M-scr}

Passons maintenant à la deuxième des questions posées au début de la section : M-scr est-il obligatoire ou optionnel ? Le déplacement d'un élément autre que le sujet est directement observable, car il se fait par-dessus le sujet, entraînant ainsi un changement important dans l'ordre des mots. En revanche, le M-scr du sujet n'étant pas aussi transparent, il n'est pas facile de savoir s'il s'est appliqué ou pas dans des phrases comme (10a) ou (11a) où le sujet précède l'objet. Mais la distribution de certains adverbes en coréen suggère, comme nous le verrons plus en détail dans ce qui suit, que M-scr s'est appliqué au sujet dans ces exemples. Il sera alors tentant de poser que M-scr s'applique toujours, quelle que soit la position occupée par le sujet en structure de surface.

Alors que dans des langues comme le français, bon nombre d'adverbes figurent normalement en position initiale de VP (ou $v \mathrm{P}){ }^{17}$, en coréen, beaucoup d'adverbes peuvent apparaître un peu partout dans la phrase comme le montre l'exemple suivant où (I)- (IV) sont les positions pour les adverbes :

(I) Pierre-ka (II) Marie-lîl (III) Paris-e (IV) ponäs'ta

-Nom -Acc -Loc envoya

'Pierre a envoyé Marie à Paris.'

La particularité du coréen est que la majorité des adverbes peuvent figurer dans toutes les positions possibles à gauche du verbe, en l'occurrence (I)- (IV) : «cacu» (souvent), «p'alli » (vite), «punmyônghi» (sûrement), « amato » (sans doute), « ôce » (hier), etc. En coréen aussi, toutefois, il existe quelques adverbes qui tendent à apparaître uniquement dans une ou deux positions. Il s'agit d'adverbes d'intensité tels que «seke » (fort), «yakhake» (doucement), «manhi» (beaucoup), «k'îke » (grandement), etc. Ces adverbes figurent, paraît-il, de préférence en position initiale de $v \mathrm{P}$ ou de $\mathrm{VP}$, comme l'illustrent les exemples suivants : 

a. cwi-ka k'îke/seke/yakhake koyangi-lîl mulôs'ô
rat-Nom Adv chat-Acc mordit
'Le rat a mordu de manière importante/fort/doucement le chat.'
b. ?? k'îke/seke/yakhake cwi-ka koyangi-lîl mulôs'ô
Adv rat-Nom chat-Acc mordit
c. (?)koyangi-lîl k'îke/seke/yakhake cwi-ka mulôs'ô
chat-Acc Adv rat-Nom mordit

Compte tenu de leur distribution dans d'autres langues, il serait raisonnable d'admettre que ces adverbes ne peuvent être engendrés hors de $v \mathrm{P}$. Ceci posé, on pourrait supposer qu'en (c), le sujet est en Spec de $v \mathrm{P}$ comme l'illustre la structure suivante : ... [vP Adv DPsujet... La (relative) grammaticalité de (c) suggère donc que la montée du sujet observée dans des langues comme le français ou l'anglais n'est pas obligatoire en coréen : si elle l'était dans cette langue, on s'attendrait à la marginalité de (c). Le contraste entre (a) et (b) ${ }^{18}$ montre d'autre part que le sujet ne peut se trouver sous $v \mathrm{P}$ lorsqu'il précède l'objet. Notons que le déplacement $\mathrm{du}$ sujet par-dessus l'adverbe en (a) ne peut être une instance de la montée du sujet : alors que celle-ci n'est pas obligatoire en coréen comme je l'ai supposé plus haut, la marginalité de (b) suggère précisément que le déplacement en question est obligatoire.

On est donc tenté de penser que le contraste entre (a) et (b) est dû au caractère obligatoire de M-scr ou au moins à une tendance forte à M-scr. Si M-scr s'applique à un élément autre que le sujet, cet élément sera hors de $v \mathrm{P}$, donc à gauche des adverbes qui eux restent sous $v \mathrm{P}$ [voir (c)]. Sinon, M-scr s'appliquera au sujet et celui-ci se trouvera hors de $v \mathrm{P}$ [voir (a)]. Quant à la marginalité de (b), on pourrait dire que c'est une phrase où M-scr ne s'est pas appliqué du tout : ni à l'objet parce que celui-ci est précédé du sujet, ni au sujet parce que l'adverbe le précède. D'où sa marginalité. Celle-ci serait mystérieuse si M-scr était optionnel.

\subsection{M-scr et la reconstruction}

Il a été noté dans la littérature que dans les phrases comme la suivante, le sujet peut à la fois avoir la portée sur toute la phrase, et la portée uniquement sur la proposition infinitive :

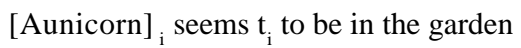

Cette ambiguïté s'explique facilement en termes de reconstruction (ou redescente). Les exemples suivants qui sont présentés par Basilico (1998) montrent également que la reconstruction est possible en cas de mouvement-A :

a. A house was built out of each pile of rubble.

b. A bale of hay was loaded onto each truck.

c. A letter was sent to every Congressperson. 
Ces phrases sont ambiguës : chacune des deux expressions quantifiées peut avoir la portée large. En permettant la reconstruction en cas de mouvement-A, on s'attend correctement à cette ambiguïté. J'admets donc que la reconstruction est en général possible en cas de mouvement-A.

Il ne semble pas en aller de même lorsqu'il s'agit d'un mouvement-A'. Saito (1989) soutient, en considérant des exemples comme le suivant, que le mouvement-A' a pour effet de fixer la portée de l'élément déplacé :

$$
\left[_{\mathrm{CP}} \text { [Which sculpture }\right]_{\mathrm{i}} \text { do }\left[_{\mathrm{IP}} \text { you know }\left[_{\mathrm{CP}} \text { that Bill carved } \mathrm{t}_{\mathrm{i}}\right]\right]
$$

Si en (16) la reconstruction était possible pour l'élément Wh-, cette phrase serait ambiguë, mais la lecture avec la portée étroite pour l'élément Wh- est totalement exclue dans cette phrase. Saito en conclut que la reconstruction est impossible en cas de mouvement-A'.

Les exemples suivants semblent indiquer que M-scr est un mouvement-A' dans la mesure où il ne permet pas la reconstruction :
a. $[=(7)]$. motu-ka ôt'ôn salam-îl mannas'ta tous-Nom quelqu'un-Acc rencontra
'Tout le monde a rencontré quelqu'un.'
b. ôt'ôn salam-îl ${ }_{i} \quad$ motu-ka t $t_{i}$ mannas'ta quelqu'un-Acc tous-Nom rencontra

(17a) est une phrase ambiguë alors qu'en (17b), il est difficile d'assigner une interprétation non spécifique au QP faible « $̂ t$ 'ôn salam-îl ». Si en (17b), l'objet direct « $\hat{o} t^{\prime} \hat{o n}$ salam-îl » pouvait se reconstruire dans sa position d'origine, alors les deux phrases auraient exactement la même structure en FL, et on s'attendrait à l'ambiguïté de cette phrase. Mais comme celle-ci n'est pas ambiguë, il serait tentant de considérer qu'il n'y pas de reconstruction dans les constructions à scrambling en coréen.

Notons qu'il existe un point de vue opposé à celui qui vient d'être présenté. Certains auteurs soutiennent, en se fondant essentiellement sur les faits de liage, que la reconstruction est possible pour l'élément déplacé par M-scr, et donc que celui-ci doit être considéré comme un mouvement-A. Par exemple, Lee (1991) présente des exemples du genre (18) :

$$
\begin{gathered}
\text { [pro }_{\mathrm{i}} \text { îimu }_{-1} \hat{l i l}_{\mathrm{j}} \quad \text { [nukuna-ka }_{\mathrm{i}} \mathrm{t}_{\mathrm{j}} \\
\text { devoir-Acc tous-Nom } \\
\begin{array}{c}
\text { chungsilhi ihänghäs'ta } \\
\text { fidèlement accomplit }
\end{array} \\
\text { 'Tout le monde a fidèlement accompli son devoir.' }
\end{gathered}
$$

En effet, si en (18), la reconstruction ne s'applique pas à l'élément déplacé, « pro » ne pourra être lié par «nukuna $(-k a)$ ». Ainsi, en prenant au sérieux ces faits de liage, on serait tenté de considérer M-scr comme un mouvement-A puisque la reconstruction y est possible. Mais compte tenu de la portée des QP 
dans les exemples $(17 \mathrm{a}, \mathrm{b})$, force est de poser le contraire, à savoir que M-scr est un mouvement-A'qui ne permet pas la reconstruction.

Pour réconcilier ces deux points de vue qui semblent a priori contradictoires, je suppose qu'en (18) l'élément en tête de phrase est en fait focalisé, et que c'est le sujet «nukuna-ka» qui est déplacé par M-scr ${ }^{19}$. Ainsi, j'admets que dans des langues comme le coréen, au moins deux éléments peuvent se trouver hors de $v \mathrm{P}$ en syntaxe visible : le focus et l'élément déplacé par M-scr. Par ailleurs, je suppose, à la suite de Diesing, que le focus doit se (re)trouver sous VP en FL ${ }^{20}$. On s'attend alors à la reconstruction de « [pro îimu]-lîl » dans sa position d'origine sous VP en (18), et donc à la grammaticalité de cette phrase ${ }^{21}$. Cela dit, compte tenu de l'absence d'ambiguïté en (17b), il faudrait admettre que dans des langues comme le coréen, la focalisation ne s'applique pas de manière identique à toutes les expressions : si la focalisation pouvait s'appliquer à «ôt'ôn salam-îl », (17b) serait ambigu ${ }^{22}$. D'autre part, il serait nécessaire de distinguer la focalisation et M-scr : alors que celui-ci est obligatoire, les données montrent qu'en coréen, celle-là est optionnelle en syntaxe explicite.

En conclusion, je considère que les deux opérations M-scr et la focalisation sont possibles dans des langues comme le coréen, et qu'à la différence de la première, la seconde permet la reconstruction. Une autre différence entre ces deux opérations est que l'une est obligatoire, alors que l'autre est optionnelle. Par conséquent, une phrase donnée peut être ambiguë du point de vue structural : plus précisément, l'élément en tête de phrase peut s'analyser ou comme le focus ou comme l'élément déplacé par M-scr.

\section{Portée des QP dans diverses constructions en coréen}

Rappelons-nous d'abord les idées principales de Diesing (1992) que j'adopte ici :

(i) les QPdoivent être divisés en QP forts et QPfaibles,

(ii) les QP forts doivent obligatoirement se trouver hors de $v \mathrm{P}$ (souvent par le biais de QR), alors que les QPfaibles peuvent éventuellement rester sous $v \mathrm{P}$ en FL,

(iii) les traces sont invisibles dans le calcul de la portée des QP.

D'autre part, en ce qui concerne M-scr en coréen, on a établi dans la dernière section les points suivants :

(iv) M-scr concerne tout élément non prédicatif : l'objet, le circonstant, et même le sujet,

(v) Dans une proposition à temps fini, un parmi les éléments non prédicatifs doit obligatoirement être déplacé par $\mathrm{M}$-scr vers une position hors de $v \mathrm{P}$,

(vi) M-scr ne permet pas la reconstruction. 
Je démontrerai dans ce qui suit que les observations ci-dessus permettent d'expliquer convenablement les faits de portée des QPdans diverses constructions à quantifieurs multiples en coréen ${ }^{23}$.

\subsection{Construction transitive simple}

Soit les exemples suivants :
a. [=(6)]. ôt'ôn salam-i motu-lîl mannas'ta quelqu'un-Nom tous-Acc rencontra
'Quelqu'un a rencontré tout le monde.'
b. motu-lîl ${ }_{i}$ ôt'ôn salam-i $t_{i}$ mannas'ta tous-Acc quelqu'un-Nom rencontra

La phrase (a) est sans ambiguïté, alors que (b) est une phrase ambiguë. M-scr étant défini comme un déplacement obligatoire vers une position hors de $v \mathrm{P}$ [voir (v)], les déplacements physiques dans ces exemples pourront être représentés comme suit :
a. $\quad\left[_{\mathrm{IP}} \text { [ôt'ôn salam-i }\right]_{\mathrm{i}}\left[_{v \mathrm{P}} \mathrm{t}_{\mathrm{i}}\right.$ motu-lîl mannas'ta $]$
b. $\left[_{\mathrm{IP}}[\text { motu-lîl }]_{\mathrm{i}}\left[_{v \mathrm{P}}\right.\right.$ ôt'ôn salam-i $\mathrm{t}_{\mathrm{i}}$ mannas'ta $]$

Je suppose d'autre part que «motu» et «ôt'ôn salam» sont respectivement un QP fort et un QP faible dans les termes de Diesing (1992) ou de Milsark (1974) [voir (i)], comme le laissent entendre leurs traductions « tous/tout le monde » et « quelqu'un ». L'objet «motu-lîl » devrait donc se déplacer obligatoirement en FL en (a). D'autre part, dans la même phrase, comme la reconstruction n'est pas permise dans les contructions à M-scr [voir (vi)], le sujet «ôt'ôn salam-i » restera hors de $v \mathrm{P}$. Par conséquent, une interprétation présuppositionnelle devra également lui être attribuée : il s'agit alors d'un individu inconnu mais spécifique au sens où son existence est présupposée par le locuteur. Les deux QP relevant ainsi uniquement de la présupposition, on s'attend correctement à l'absence d'ambiguïté dans cette phrase. En revanche, en (b), le sujet «ôt'ôn salam-i » qui se trouve sous $v \mathrm{P}$ en syntaxe visible pourra éventuellement se déplacer hors de $v \mathrm{P}$ en FL : lorsqu'il y monte, il aura une interprétation présuppositionnelle, c'est-àdire quelqu'un de spécifique ; lorsqu'il reste sous $v \mathrm{P}$, il aura une interprétation variable. D'où l'ambiguïté de (b) ${ }^{24}$.

Considérons maintenant les constructions suivantes où le sujet est un QP fort alors que l'objet est un QP faible :
a. motu-ka ôt'ôn salam-îl mannas'ta tous-Nom quelqu'un-Acc rencontra 'Tout le monde a rencontré quelqu'un.'
b. ôt'ôn salam-îl $l_{i}$ motu-ka $t_{i}$ mannas'ta quelqu'un-Acc tous-Nom rencontra 
La phrase (a) est ambiguë, alors qu'en (b), il est difficile d'obtenir l'interprétation variable pour l'objet «ôt'ôn salam-îl ${ }^{25}$. Cette différence sémantique entre (a) et (b) est correctement prédite par mon analyse résumée en (i)-(vi). En (a), le sujet qui est un QP fort restera hors de $v \mathrm{P}$ en FL, et l'objet qui est un QP faible peut optionnellement se déplacer vers une position hors de $v \mathrm{P}$ en FL. Ainsi s'explique l'ambiguïté de (a). En (b), la reconstruction ne pouvant avoir lieu, l'objet déplacé en syntaxe visible en tête de phrase doit rester hors de $v \mathrm{P}$ même en FL. Et le sujet $\mathrm{QP}$ fort qui se trouve en syntaxe explicite en Spec de $v \mathrm{P}$ doit obligatoirement se déplacer hors de $v \mathrm{P}$ en FL. Alors, les deux QPseront uniquement dans le domaine de présupposition, et on s'attend à l'absence d'ambiguïté en (b).

Notons en passant que pour une partie considérable des locuteurs du coréen, lorsqu'on remplace, en (21b), le QP faible par un autre QP faible «nukunka » (quelqu'un), la phrase devient ambiguë. De même, lorsqu'on met «yôlô salam» (plusieurs personnes) à la place du QPfaible en question, beaucoup de locuteurs trouvent la phrase ambiguë. Ainsi, le choix de l'item lexical joue un rôle non négligeable dans l'interprétation des QP faibles. Pour rendre compte de ce fait, je suppose que le degré de focalisabilité varie selon l'item lexical, du moins s'il s'agit d'un QP faible ${ }^{26}$. Considérons l'exemple suivant :
A: Pierre-ka nuku-lîl mannas'ô ?
- Nom qui-Acc rencontra
'Qui est-ce que Pierre a rencontré ?'
B : (Pierre-ka) (?)yôlô salam/(?)nukunka/?*ôt'ôn salam- (l)îl mannas'ô - Nom plusieurs personnes/quelqu'un/quelqu'un-Acc rencontra 'C'est plusieurs personnes/quelqu'un que Pierre a rencontré.'

À la différence des autres QP faibles, «ôt'ôn salam » ne peut être utilisé dans la réponse à la question $\mathrm{A}$. Cela est dû, semble-t-il, à la différence de focalisabilité entre ces QP faibles. En d'autres termes, il serait impossible ou difficile de focaliser « $\hat{o}$ ' $\hat{o} n$ salam », alors que les deux autres QPpeuvent éventuellement se focaliser. J'admets d'autre part, suivant Diesing (1992, § 2.6), qu'à la différence du sujet engendré hors de VP, les éléments engendrés sous VP doivent se reconstruire dans leur position d'origine lorsqu'ils sont focalisés ${ }^{27}$. Ceci posé, on pourrait dire que l'ambiguïté en question chez certains locuteurs tient au fait que le QP faible en tête de phrase peut s'analyser de deux façons différentes, c'est-àdire soit comme focalisé soit comme déplacé par M-scr. Alors, ce QP faible recevra l'interprétation variable dans le premier cas, et la spécifique dans le second cas ${ }^{28}$.

\subsection{Constructions intransitives}

Voici quelques exemples de constructions intransitives en coréen :

a. motu-ka ôtinka-esô nolähäs'ta tous-Nom quelque part-Loc chanta

'Tout le monde a chanté quelque part.' 
b. ôtinka-esô motu-ka nolähäs'ta

quelque part-Loc tous-Nom chanta

La phrase (a) est ambiguë : elle peut signifier à la fois (i) «tout le monde a chanté dans un endroit spécifique, par exemple dans l'auditorium » et (ii) « A a chanté dans la salle A', B dans la salle B', C dans la salle C', ainsi de suite... ». En revanche, il est difficile d'obtenir l'interprétation (ii) pour (b). Cette différence sémantique entre les exemples ci-dessus sera correctement prédite en posant que leur structure de base est (24a) ou (24b) :

a. $\left[_{v \mathrm{P}}\right.$ motu-ka... $\left[_{\mathrm{VP}}\right.$ ôtinka-esô...

b. $\left[_{v \mathrm{P}}\right.$ ôtinka-esô $\left[_{v \mathrm{P}}\right.$ motu-ka...

Si M-scr déplace le sujet «motu-ka » hors de $v \mathrm{P}$, le $\mathrm{PP}$ «ôtinka-esô » restera sous $v \mathrm{P}$ en syntaxe visible comme le représente la structure suivante :

a. $\left[_{\mathrm{IP}}\right.$ motu-ka... $\left[_{v \mathrm{P}} \ldots\right.$ ôtinka-esô...

En FL, le PP « ôtinka-esô », QP faible, pourra optionnellement se déplacer hors de $v \mathrm{P}$ : lorsqu'il y monte, on aura l'interprétation (i); autrement, on aura (ii). D'où l'ambiguïté de (23a). Mais si c'est au PP «ôtinka-esô » que M-scr s'applique, on aura alors une structure comme la suivante :

b. $\left[_{\mathrm{IP}}\right.$ ôtinka-esô... $\left[_{v \mathrm{P}}\right.$ motu-ka...

Lorsqu'en (25b) QR s'applique au sujet QP fort, les deux QP seront dans le domaine de présupposition. On s'attend alors à l'absence d'ambiguïté en (23b).

Considérons maintenant les constructions suivantes où le sujet est un QP faible, alors que le PP est un QPfort :

a. (ôce pam) ôt'ôn salam-i motîn pang-esô cas'ta hier nuit quelqu'un-Nom tout chambre-Loc dormit 'La nuit dernière, quelqu'un a dormi dans toutes les chambres.'

b. (ôce pam) motîn pang-esô ôt'ôn salam-i cas'ta hier nuit tout chambre-Loc quelqu'un-Nom dormit

La phrase (26a), non ambiguë, se dira, par exemple, pour décrire la situation suivante : Pierre ou Jean, même si on ne sait pas lequel, a dormi de $22 \mathrm{~h}$ à $1 \mathrm{~h}$ dans la chambre $\mathrm{A}$, puis il est allé dans la chambre $\mathrm{B}$ pour y dormir jusqu'à $3 \mathrm{~h}$, enfin il s'est réveillé à $7 \mathrm{~h}$ dans la chambre C. Mais la phrase (26b), ambiguë, pourra se dire non seulement dans cette dernière situation, mais aussi lorsque Pierre, Jean et Marie ont dormi toute la nuit respectivement dans les chambres A, B et C. Je suppose que dans le premier cas, on a affaire à l'interprétation présuppositionnelle ou spécifique du QP faible «ôt'ôn salam », alors que c'est de son interprétation 
non présuppositionnelle ou variable qu'il s'agit dans le second. Il semble que ces faits soient parfaitement prédits par mon analyse. La représentation pertinente avant FLdes phrases (26a) et (26b) serait respectivement (27a) et (27b) :

$\begin{array}{ll}\text { a. } & {\left[_{\mathrm{IP}} \text { ôt'ôn salam-i... }\left[_{v \mathrm{P}} \ldots \text { motîn pang-esô... }\right.\right.} \\ \text { b. } & {\left[_{\mathrm{IP}} \text { motîn pang-esô... }\left[_{v \mathrm{P}} \text { ôt'ôn salam-i... }\right.\right.}\end{array}$

En (a), le QP « $\hat{t}$ ' $̂ n$ salam- $i$ » est hors de $v \mathrm{P}$ en syntaxe explicite, et il y restera en FL, tout simplement parce que la reconstruction est exclue pour M-scr. Par ailleurs, le QP fort «motîn pang-esô » se déplace aussi hors de $v \mathrm{P}$ en FL. Ainsi, pour (26a), les deux QP se trouveront en FL dans le domaine de présupposition, auquel cas on aura l'interprétation spécifique pour le $\mathrm{QP}$ «ôt'ôn salam- $i$ ». En revanche, en (27b), ce dernier peut à la fois rester sous $v \mathrm{P}$ et se déplacer hors de $v \mathrm{P}$; au cas où il bouge, l'interprétation de la phrase sera identique à celle de $(27 \mathrm{a})$, mais au cas où il ne bouge pas, on aura l'interprétation variable pour le $\mathrm{QP}$ « $\hat{t}$ ' $̂$ n salam-i ». D'où l'ambiguïté de (27b).

Il est généralement admis que les verbes intransitifs comprennent deux groupes : les inergatifs et les inaccusatifs. Les phrases que nous venons de voir sont en fait des exemples pour les inergatifs. Considérons donc quelques exemples pour les inaccusatifs :
a. motu-ka ôtinka-lo t'ônas'ta
tous-Nom quelque part-vers partit
'Tout le monde est parti quelque part.'
b. ôtinka-lo motu-ka t'ônas'ta quelque part-vers tous-Nom partit
a. ôt'ôn salam-i motîn nara-e kas'ta quelqu'un-Nom tout pays-Loc alla 'Quelqu'un est allé dans tous les pays.'
b. motîn nara-e ôt'ôn salam-i kas'ta tout pays-Loc quelqu'un-Nom alla

La phrase (28a) est ambiguë : « ôtinka-lo», QP faible, peut être spécifique, mais il peut aussi être non spécifique, c'est-à-dire variable. Il en est de même pour (29b) : le QP faible «ôt'ôn salam-i » peut à la fois être spécifique et non spécifique. Dans les deux autres exemples, on n'a qu'une interprétation pour le QP faible : celle de présupposition. Les représentations pertinentes après M-scr pour les constructions inaccusatives $(28 \mathrm{a}, \mathrm{b})$ et $(29 \mathrm{a}, \mathrm{b})$ seraient respectivement :
a. $\left[_{\mathrm{IP}}\right.$ motu-ka... $\left[_{\mathrm{VP}}\right.$ ôtinka-lo...
b. $\left[_{\mathrm{IP}}\right.$ ôtinka-lo... [ $\left[_{\mathrm{VP}}\right.$ motu-ka...
c. $\left[_{I P}\right.$ ôt'ôn salam-i... $\left[_{V P}\right.$ motîn nara-e...
d. $\left[_{I P}\right.$ motîn nara-e... $\left[_{V P}\right.$ ôt'ôn salam-i... 
Ces structures sont comparables aux structures $(25 a, b)$ et $(27 a, b)$, à ceci près que $\nu \mathrm{P}$ dans celles-ci est remplacé par VP dans celles-là. Mais il semble que cette dernière différence n'affecte en rien les faits de portée des QP dans les constructions considérées. On peut donc expliquer l'ambiguïté et l'absence d'ambiguïté dans les constructions inaccusatives exactement comme on l'a fait plus haut pour les constructions inergatives ${ }^{29}$.

\subsection{Construction passive}

Examinons maintenant si mon analyse fait des prédictions correctes pour la construction passive. Soit les exemples suivants :
a. motu-eîihä ôt'ôn salam-i chotätöôs'ta
tous-par quelqu'un-Nom fut invité
'Quelqu'un a été invité par tout le monde.'
b. ôt'ôn salam-i motu-en̂ihä chotätöôs'ta quelqu'un-Nom tous-par futinvité

La phrase (a) est ambiguë. Elle peut signifier (i) que tout le monde a invité la même personne dont le locuteur ne veut (ou peut) pas préciser l'identité pour une raison ou pour une autre, et (ii) que chacun a invité son ami : A a invité $\mathrm{X}, \mathrm{B}$ a invité Y, C a invité Z, ainsi de suite. Mais en (b) le QPfaible «ôt'ôn salam- $i$ » ne peut avoir que l'interprétation présuppositionnelle, c'est-à-dire (i) ${ }^{30}$. Vu l'ordre des mots en (a), je suppose que dans cette phrase, le PP est déplacé par M-scr, et par conséquent, restera dans le domaine de présupposition en FL. Mais le sujet, QP faible, peut optionnellement monter par QR en FL. On s'attend donc à l'ambiguïté de cette phrase. En revanche, comme en (b), c'est le QPfaible qui est déplacé par M-scr, on s'attend à l'absence d'ambiguïté dans cette phrase. Considérons maintenant les phrases suivantes où le sujet est un QP fort :
a. ôt'ôn salam-eîihä motu-ka chotätöôs'ta quelqu'un-par tous-Nom fut invité 'Tout le monde a été invité par quelqu'un.'
b. motu-ka ôt'ôn salam-eîihä chotätöôs'ta tous-Nom quelqu'un-par fut invité

Je suppose, compte tenu de l'ordre des mots, qu'en (b), le QP faible n'est pas déplacé par M-scr. On s'attend donc à l'ambiguïté de cette phrase. En revanche, en (a), le QP faible étant déplacé par M-scr, on s'attend à l'absence d'ambiguïté. Ces prédictions semblent correctes : les deux phrases peuvent se dire lorsque A, $\mathrm{B}, \mathrm{C}$ sont invités par une seule et même personne, alors que seule la phrase (b) peut se dire dans la situation où $\mathrm{A}, \mathrm{B}, \mathrm{C}$ sont invités respectivement par $\mathrm{X}, \mathrm{Y}, \mathrm{Z}$. 


\section{Quelques spéculations}

Ainsi, il a été démontré dans la dernière section que la théorie de Diesing (1992), combinée avec quelques observations indépendantes sur le coréen et surtout la théorie de la phrase proposée par Chomsky (1995), rend possible l'analyse formelle et adéquate des faits de portée des QPen coréen. Mais au cours de la démonstration, j'ai dû réinterpréter quelques notions de Diesing en termes minimalistes : par exemple, j'ai remplacé « $\mathrm{VP} »$ de Diesing par « $v \mathrm{P} »$.

Je suppose qu'un autre réajustement est nécessaire : il s'agit de QR. Car dans la Théorie Minimaliste, tout déplacement est la conséquence de Attirer-F, alors que QR, tel qu'il est initialement formulé, s'applique de lui-même, sans être déclenché par une autre catégorie. Mais la théorie de QR telle qu'elle est présentée dans Diesing (1992) semble plus apte à s'incorporer au modèle minimaliste. D'un point de vue minimaliste, on pourrait supposer que comme tous les autres déplacements, QR est déclenché par le trait [Présupposé] de I, et que QR, invisible, n'est pas un déplacement de la catégorie entière, mais du trait nécessaire à la vérification du trait [Présupposé] de $\mathrm{I}^{31}$. Ce dernier trait ne semble pas être de nature morphologique, alors que dans la Théorie Minimaliste, seuls les traits morphologiques peuvent déclencher un déplacement. Mais étant donné que QR est une notion de toute façon nécessaire pour rendre compte de nombreux phénomènes linguistiques, il serait toujours envisageable de modifier la Théorie Minimaliste de manière à incorporer $\mathrm{QR}$.

Notons que M-scr est également une notion difficile à réconcilier avec la Théorie Minimaliste, parce qu'il est souvent considéré comme un déplacement gratuit ou optionnel. M-scr est comparable à $\mathrm{QR}$ dans la mesure où tous les deux sont des déplacements hors de $v$ P. En fait, rien ne semble empêcher de les considérer comme des déplacements dans le domaine de vérification de I ${ }^{32}$. Mais étant abstrait, QR serait le déplacement du seul trait de QP nécessaire à vérifier le trait [Présupposé] de I, alors que M-scr, physique, est le déplacement d'une catégorie entière. Une autre différence entre les deux déplacements serait que QR ne concerne que les QP, alors que M-scr concernerait toute catégorie non prédicative. Pour rendre compte de ces faits, on pourrait faire l'hypothèse, par exemple, que I contient également un trait qui est distinct de [Présupposé], et assez « fort » dans certaines langues pour déclencher un déplacement physique obligatoire. L'examen cursif de données élémentaires semble suggérer que le trait en question est [Topique] ${ }^{33}$, mais pour déterminer sa nature exacte, il faudrait une étude beaucoup plus rigoureuse de la sémantique des constructions à M-scr. Je laisse cette tâche pour de futurs travaux.

\section{NOTES}

* This paper was accomplished with Research Fund provided by Korea Research Foundation, Support for Faculty Research Abroad. Ce travail a été présenté oralement en novembre 1998 dans le Séminaire commun UP 8-UP3. Je remercie Anne Zribi-Hertz, Georges 
Rebuschi, Jean-Yves Pollock, Alain Rouveret et deux évaluateurs anonymes de Recherches linguistiques de Vincennes pour leurs commentaires précieux, et Makoto Kaneko de m'avoir aidé pour les données japonaises.

1. L'ambiguïté de (1a) n'est pas incontestée comme me le fait remarquer un évaluateur anonyme. Mais cette question ne semble pas cruciale dans mon analyse. Je procéderai donc, pour être concret, comme si cette ambiguilté était incontestée.

2. L'analyse qui va être présentée pourrait s'appliquer assez facilement au japonais aussi. Mais ces deux langues ne semblent pas se comporter exactement comme le chinois en ce qui concerne la portée des quantifieurs (voir $§ 2.2$ de ce travail). Dans ce travail, en me concentrant essentiellement sur ces deux premières langues, je ne tiendrai pas compte des faits en chinois. Par ailleurs, je ne parlerai des données japonaises que lorsque celles-ci représentent une différence significative avec les données coréennes.

3. Tout au long du présent travail, la traduction française des exemples coréens ne sera qu'approximative, et des précisions supplémentaires seront fournies si nécessaire.

4. Il semble que les locuteurs japonais ont tendance à ne pas voir d'ambiguité dans la traduction japonaise de (7), quoiqu'il existe toujours un certain nombre de locuteurs qui la trouvent ambiguë. Je reviens sur cette question en fin de $\S 4.1$ et dans la note 28.

5. Cette idée est également admise dans Aoun et Li (1993) et Hornstein (1995), entre autres.

6. «Materiel from VP is mapped into the nuclear scope. Material from IP in mapped into a restrictive clause. » [Diesing $(1992,10)$.]

7. En reprenant les termes de Diesing (1992) tels quels, j'ai noté «VP» dans le texte. Mais je considère que dans les termes minimalistes, le VPde Diesing correspond souvent à $v \mathrm{P}$.

8. Mais je suppose que la reconstruction est impossible ou très difficilement permise en cas de M-scrambling (voir $§ 3.3$ ).

9. Les termes exactes de Milsark et de Diesing sont respectivement « déterminant » et « quantifieur », mais j'adopte le terme «QP» pour la commodité de l'exposé.

10. Suivant Diesing, je considérerai ici les indéfinis comme quantifieurs. Mais je ne parlerai pas des expressions comme «tu salam» (deux personnes), car celles-ci peuvent fonctionner à la fois comme des définis et comme des indéfinis dans des langues comme le coréen qui n'ont pas d'articles.

11. Diesing décrit les trois lectures associées aux représentations $(9 \mathrm{a}-\mathrm{c})$ de la manière suivante. Soit la situation dans laquelle les violoncellistes participant à un concours doivent jouer chacun quelques variations devant le jury. Le concours peut se dérouler de trois façons différentes en ce qui concerne le choix de variations par les violoncellistes. Le premier scénario, qui est représenté par (9c), est que les violoncellistes doivent tous jouer les mêmes variations choisies préalablement par le jury. Dans ce scénario, la portée large sera assignée au DPobjet, et ce dernier sera spécifique. Le deuxième est que chaque violoncelliste doit choisir quelques-unes des variations figurant sur la liste préétablie par le jury. Alors, le DP objet aura une portée étroite, mais recevra une interprétation spécifique en ce sens qu'il fait partie de la liste "présupposée". Cette situation est représentée par (9a). Le dernier scénario, qui est représenté par (9b), est que chaque violoncelliste doit improviser, pour le concours, des variations quelconque, sans qu'une liste en soit proposée par le jury. Dans ce cas, le DPobjet aura une portée étroite, et les variations jouées varieront d'un musicien à l'autre, c'est-à-dire qu'une interprétation variable est assignée au DPobjet.

12. Il est courant de distinguer trois types de scrambling dans des langues comme le japonais ou le coréen : $\mathrm{S}$ (hort)-scrambling, $\mathrm{M}$ (iddle)-scrambling et $\mathrm{L}$ (ong)-scrambling. Le premier désigne le déplacement au sein de VP. Par conséquent, même lorsqu'il s'applique, le sujet engendré hors de VP reste toujours en tête de phrase. Le troisième est le déplacement d'un élément de l'enchâssée à gauche du sujet de la matrice. Et le deuxième est le déplacement d'un élément de la matrice à gauche du sujet de la matrice. Dans ce travail, je ne tiens compte que de Mscrambling. 
13. Je ne prends pas ici en considération les éléments dominés par un DP, car celui-ci constitue, en coréen, une barrière (quasi)infranchissable pour les éléments qu'il domine. J'adopte par ailleurs l'idée couramment admise que les adverbes ne se déplacent pas, alors qu'ils peuvent être engendrés dans plus d'une positions syntaxiques.

14. Que M-scr soit une adjonction à IP, ou un déplacement dans son Spec ou vers une postion plus élevée, n'affecte en rien mon analyse, mais je considère pour le moment, pour être concret, que M-scr est une adjonction à IP. Je reviens plus tard sur cette question (voir $\S 5$ et note 32 ).

15. K. Tateishi est un des rares linguistes qui supposent le scrambling du sujet [voir Tateishi $(1994, \S 8.3)]$.

16. Je considère toutefois que la focalisation et M-scr sont deux processus distincts (voir § 3.3).

17. Voir entre autres Pollock (1989).

18. L'acceptabilité de (b) s'améliore considérablement lorsqu'une pause nette est mise après l'adverbe. Je suppose que la pause sert à marquer le focus, et que la focalisation de l'adverbe d'intensité est acceptée dans une certaine mesure en coréen.

19. Par conséquent, la structure pertinente de (18) sera quelque chose comme la suivante : $\mathrm{F}_{\text {Focp }}$ [pro ${ }_{\mathrm{i}}$ î̀mu $]-1 \hat{1} \mathrm{l}_{\mathrm{j}}\left[_{\mathrm{IP}} \text { [nukuna-ka }\right]_{\mathrm{i}}\left[_{v \mathrm{P}}\right.$ chungsilhi $\mathrm{t}_{\mathrm{i}} \mathrm{t}_{\mathrm{j}} \ldots$

20. Une idée semblable est proposée par Valduví (1992) dans un contexte un peu différent.

21. Un évaluateur anonyme note que si un adverbe d'intensité peut apparaître entre le sujet et le verbe dans des phrases comme (18), cela renforcera mon analyse qui consiste à poser, pour des phrases en question, deux déplacements hors de $v \mathrm{P}$, à savoir la focalisation de l'objet et M-scr du sujet. Les phrases coréennes suivantes semblent en effet militer en faveur de mon analyse :

(i) [pro îimu]-lîl ${ }_{j}$ [nukuna-ka $]_{I}$ k'îke/yakkan t tînghanhihäs'ta

devoir-Acc tous-Nom grandement/un peu négligea

('Tout le monde a négligé beaucoup/un peu son devoir.')

(ii) ??[pro îimu $]-l i ̂ 1_{\mathrm{j}}$ k'îke/yakkan [nukuna-ka $]_{\mathrm{i}} \mathrm{t}_{\mathrm{j}}$ tînghanhihäs'ta

devoir-Acc grandement/un peu tous-Nom négligea

(iii) [sul]-îl $1_{\mathrm{j}}$ [nukuna-ka $]_{\mathrm{i}}$ manhi/yakkan $\mathrm{t}_{\mathrm{i}} \mathrm{t}_{\mathrm{j}}$ masiôs'ta

alcool-Acc tous-Nom beaucoup/un peu but

('Tout le monde a bu beaucoup/un peu d'alcool.')

(iv) (?)[sul]-îl $1_{j}$ manhi/yakkan [nukuna-ka] $t_{j}$ masiôs'ta

alcool-Acc beaucoup/un peu tous-Nom but

Le contraste entre (i) et (ii) semble indiquer que le sujet doit s'extraire de $\nu \mathrm{P}$ si l'objet en tête de phrase est un focus, et donc doit se reconstruire sous $v$ P. D'autre part, le contraste entre (iii) et (iv) suggère que le sujet peut rester sous $v \mathrm{P}$ si le DP objet n'est pas focalisé, et par conséquent n'a pas à se reconstruire dans sa position d'origine.

22. Je suppose en fait que le degré de focalisabilité de chaque QP faible varie selon l'item lexical (voir la discussion en fin de $\$ 4.1$ ).

23. La théorie de Diesing semble adéquate pour expliquer la portée des QP dans les propositions simples, mais les faits sont beaucoup plus compliqués dans les propositions complexes, et pour en rendre compte, il faudrait des principes supplémentaires comme ceux que propose Yang (1993), par exemple. Dans ce travail, je ne m'occuperai que des propositions simples. D'autre part, je ne parlerai pas ici de la construction ditransitive, en partie parce que les jugements des locuteurs pour les exemples de celle-ci varient beaucoup trop probablement à cause de la complexité de sa structure argumentale (avec trois arguments), en partie parce que je ne connais pas l'analyse de cette construction cohérente et surtout pleinement compatible avec le Minimalisme, modèle auquel je me réfère.

24. On notera qu'en (b), l'objet en tête de phrase restera hors de $v \mathrm{P}$ même en FL, non seulement parce que c'est un QP fort, mais parce que la reconstruction n'est pas permise pour l'élément déplacé par M-scr. 
25. Il se pourrait que dans des phrases comme (21a) ou (21b), on puisse distinguer deux interprétations spécifiques du QP faible « ôt'ôn salam » comme Diesing le fait à propos de la phrase (8) [voir (9a) vs. (9c)]. Mais pour être concret, dans ce travail, je ne prends en considération que la distinction entre la spécifique et la variable.

26. J'entends par le focus « la nouvelle information de la phrase que le locuteur veut fournir à l'allocutaire ». Il semble que le focus est mis en accent phonétiquement dans beaucoup de langues. Toutefois, l'accentuation phonétique étant réalisée et perçue souvent de manière peu nette dans des langues comme le coréen, je ne la considère pas comme un critère nécessaire pour définir le focus. Je suppose donc que la focalisation est avant tout un phénomène sémanticopragmatique. De ce point de vue, la focalisabilité d'un item lexical tiendrait le plus souvent à son contenu sémantique. Par conséquent, toute expression référentielle pourrait en principe se focaliser. Le fait que certains QP faibles soient difficiles à focaliser serait sans doute dû à leur caractère non (ou peu) référentiel. Mais j'ignore pourquoi certaines expressions peuvent se focaliser plus facilement que d'autres, et quel(s) est (ou sont) précisément le(s) trait(s) de celleslà qui rend(ent) possible leur focalisation.

27. La reconstruction peut donc s'appliquer non seulement à l'objet, mais aussi au sujet de l'inaccusative, de la passive, etc. En revanche, si le sujet agentif est toujours engendré hors de VP comme le soutient Chomsky (1995), dans l'esprit de Hale et Keyser (1993) le sujet de la transitive ou de l'inergative devra se trouver en FL dans le domaine présuppositonnel même lorsqu'il se focalise et par la suite se reconstruit. Par conséquent, je suppose que la focalisation du sujet agentif n'a aucun effet sur l'ambiguïté de la phrase.

28. La traduction japonaise de (21b) avec l'expression « dareka» (quelqu'un) pour le QPfaible est le plus souvent considérée comme ambiguë dans la littérature [voir Miyagawa, 1997, (31), Yatsushiro, 1996, (4a) entre autres]. Cela s'expliquera lorsqu'on suppose que le QP faible «dareka » peut très facilement se focaliser et donc se reconstruire en japonais. En outre, du fait que les locuteurs japonais ont tendance à ne pas voir d'ambiguité dans la traduction japonaise de (21a) [voir la note 3], je fais l'hypothèse que « dareka» tend fortement à se focaliser, sauf lorsqu'il est déplacé par M-scr, opération qui empêche la reconstruction. Dans cette hypothèse, comme en (21a), M-scr ne s'y est pas appliqué, « dareka» risquera fort de se focaliser et de rester sous VP.

29. Il semble toutefois que les deux constructions intransitives doivent être analysées différemment lorsque le sujet est un QP faible susceptible de se focaliser (voir la discussion en fin de $\S 4.1$ ). Les locuteurs qui voient l'ambiguïté en (i) sont plus nombreux que ceux qui la voient en (ii), semble-t-il :

(i) nukunka-ka motîn nara-e kas'ta quelqu'un-Nom tout pays-Loc alla

(ii) nukunka-ka motîn pang-esô norähäs'ta quelqu'un-Nom toute pièce-Loc chanta

Cela s'explique lorsqu'on admet que le sujet est engendré sous VP dans la construction inaccusative, et en Spec de $v \mathrm{P}$ dans l'inergative : seul le sujet de l'inaccusative pourra se reconstruire.

Si le QP japonais «dareka» (quelqu'un) se focalise encore plus facilement que le QP coréen «nukunka » (voir la note précédente), on s'attend à ce que l'équivalent japonais de (i) soit encore plus ambigu que la phrase coréenne, et que celui de (ii) soit non ambigu. D'après Yatsushiro (1996, 318-319), cette prédiction est correcte. Voici les exemples qu'elle présente :

(iii) dareka-ga dono-heya-ni-mo tuita quelqu'un-Nom tout-pièce-Loc-aussi arriva 'Quelqu'un est arrivé dans toutes les pièces.'

(iv) dareka-ga dono-isu-ni-mo suwatta quelqu'un-Nom tout-chaise-Loc-aussi s'assit 'Quelqu'un s'est assis sur toutes les chaises.' 
L'auteur considère que (iii) est ambigu, alors qu'en (iv), seule la lecture spécifique peut être assignée au QPfaible «dareka-ga».

30. Certains locuteurs trouvent la phrase ambiguë lorsque le sujet est remplacé par un autre QP faible plus focalisable comme le prédit mon analyse (voir la discussion en fin de $§ 4.1$ et la note précédente). De même, le QP japonais «dareka » se focalisant facilement, on s'attend à l'ambiguïté de l'équivalent japonais de (32b). Yatsushiro suppose que l'exemple suivant est effectivement ambigu :

(i) dareka-ga dono-mati-ni-mo okur-are-ta

quelqu'un-Nom tout-ville-Loc-aussi fut envoya

'Quelqu'un a été envoyé dans toutes les villes.'

31. Dans un esprit similaire, Yang (1993) suppose que Temps a le trait [Opérateur], et que QR est motivé pour la vérification de ce dernier trait. Il soutient par ailleurs que QR doit être considéré comme un déplacement de D, non pas de DP.

32. Si en coréen, le trait [D] de I est faible, la montée du sujet pour la vérification casuelle n'aura pas lieu en syntaxe visible, et en FL, les traits formels du sujet seront attirés directement sur I, non pas dans son Spec. Ainsi, en coréen, le Spec de IPne servira jamais de site d'arrivée pour le sujet qui monte pour des raisons casuelles, et par conséquent, il n'y aura aucune raison de considérer cette position comme une position-A. Il se pourrait donc qu'en coréen, cette position, en tout cas si elle existe dans cette langue, puisse servir de site d'arrivée pour M-scr, que ce dernier soit un mouvement-A ou un mouvement-A'. Dans un contexte différent, Déprez (1994) propose une analyse qui consiste à dire que la position qui sert de site d'arrivée pour le scrambling en allemand peut avoir à la fois des propriétés de position-Aet de position-A'.

33. Saito (1985), Lasnik \& Saito (1992) entre autres, supposent que le scrambling en japonais resemble beaucoup à la topicalisation en anglais. Miyagawa (1997) soutient que M-scr est motivé ou bien pour la vérification du Cas, ou bien pour la focalisation. Mais il attribue à cette dernière une définition semblable à celle qu'on donne couramment au topique [voir Miyagawa (1997, p. 10, la note 17)]. Or M-scr ne déplace pas uniquement les éléments portant le marqueur de topique, et ces derniers restent souvent dans leur position d'origine. Par ailleurs, il semble exister des contextes où le DP portant le marqueur de topique ne peut remplacer le DP accusatif ou nominatif déplacé par M-scr et vice versa. Il faudrait donc être très prudent lorsqu'on veut rapprocher M-scr de la topicalisation.

\section{RÉFÉRENCES}

Aoun, J. ; LI, Y.A. (1989). Constituency and scope. Linguistic Inquiry 20 : 141-147.

Aoun, J. ; LI,Y. A. (1993). Syntax of scope, Cambridge, Massachusetts: MIT Press.

BASILICO, D. (1998). Object position and predication forms. Natural Language and Linguistic Theory $16: 541-595$.

Сномsку, N. (1993). A minimalist program for linguistic theory. Dans Hale \& Keyser (eds), The view from Building 20. Cambridge, Massachussets: MIT Press.

Chomsky, N. (1995). The Minimalist Program. Cambridge, Massachussets: MITPress.

DÉPREZ, V. (1994), Parameters of object movement. Dans Corver \& Riemsdijk (eds), Studies on scrambling. Berlin-New York: Mouton de Gruyter.

DIESING, M.(1992). Indefinites, Cambridge, Massachusetts : MITPress.

Hale, K. ; Keyser, S. J. (1993). On argument structure and the lexical expression of syntactic relations, Dans Hale \& Keyser (eds), 1993. The view from Building 20. Cambridge, Massachussets : MITPress.

HoRnstein, N. (1995). Logical Form : The grammar of Logical Form from GB to minimalism, Oxford: Blackwell. 
HuAnG, C.-T. J. (1982). Logical relation in Chinese and the theory of grammar. Doctoral dissertation, MIT.

LasniK, H. ; SaIto, M. (1992). Move-Mo, Cambridge, Massachussets : MITPress.

LEE, Y. S. (1991). Scrambling and pronoun binding. Dans Kuno et alii, (eds), Harvard Studies in Korean Linguistics IV. Cambridge, Massachussets : Department of Linguistics, Harvard University.

MAY, R. (1977). The grammar of quantification, Doctoral dissertation, MIT.

MAY, R. (1985). Logical Form, Cambridge, Massachussets : MITPress.

MilsARK, G. (1974). Existential sentences in English. Doctoral dissertation, MIT.

MiYagawa, S. (1997). Against optional scrambling. Linguistic Inquiry $28: 1-25$.

PolLOCK, J.-Y. (1989). Verb movement, universal grammar, and the structure of IP. Linguistic Inquiry $20: 365-425$.

SAito, M. (1985). Some asymetries in Japanese and their theoritical implications. Doctoral dissertation, MIT.

SAITo, M. (1989). Scrambling as semantically vacuous A'-movement. Dans Baltin \& Kroch, (eds), Alternative conceptions of phrase structure, Chicago : University of Chicago Press.

TATEISHI, K. (1994). The syntax of «subjects», Tokyo : Kurosio Publishers.

VALDuví, E. (1992). The informational component, New York : Garland.

YANG, D. H. (1993). A minimalist approach to quantification. Studies in Generative Grammar $3.2: 311-333$.

Yatsushiro, K. (1996). Case, scope, and feature movement. Working papers in linguistics 7, University of Connecticut.

\begin{abstract}
This article aims at describing the quantifier scope in various multi-quantifier constructions in Korean. For that purpose, it adopts the theory of Diesing which offers an explaination of many quantification phenomena in different languages. Moreover, it proposes a new analysis of a movement called «M(iddle)-scrambling» in languages like Korean. For a long time, it has been thought in the literature that the subjet behaves as an exception with respect to M-scrambling. But this widely admitted idea is rejected in this paper. It is demonstrated here that M-scrambling is a movement toward a position outside $\nu \mathrm{P}$ which concerns all the non predicative phrases. This analysis of $\mathrm{M}$ scrambling, combined with the theory of Diesing, offers a correct explaination of quantification facts in Korean.
\end{abstract}

\title{
KEYWORDS
}

Quantifier scope, strong quantifier, weak quantifier, M-scrambling, focalization, reconstruction, Diesing, QR, presupposition, specific interpretation, variable interpretation, Korean, ambiguity. 